\title{
MEDIDA DA VELOCIDADE DE FLUXO NAS ARTÉRIAS CEREBRAIS UTILIZANDO ULTRA-SOM DOPPLER TRANSFONTANELA ANTES E APÓS O TRATAMENTO CIRÚRGICO DA HIDROCEFALIA
}

\author{
MARCELO CARDOSO DE ASSIS*, HELIO RUBENS MACHADO**
}

\begin{abstract}
RESUMO - Vinte e sete crianças portadoras de hidrocefalia de diversas etiologias, diagnosticadas pelo exame clínico, neurosonografia e tomografia cerebral computadorizada, foram submetidas a avaliação por ultra-som Doppler (US-Doppler) transfontanela para medida da velocidade de fluxo sanguíneo e cálculo do índice de resistência (IR) nas artérias cerebrais anteriores, médias e carótidas internas. Todas as crianças foram submetidas a avaliações no pré-operatório e no $1^{\circ}, 7^{\circ}, 30^{\circ}$ e $60^{\circ}$ dias do pós-operatório. Concluímos ser a técnica de neurosonografia e US-Doppler útil no diagnóstico da hidrocefalia, na indicação e controle das derivações liquóricas e no acompanhamento das modificações dos valores do IR comparando-os no pré e pós-operatório imediato e tardio do tratamento cirúrgico da hidrocefalia. Os resultados obtidos permitiram-nos ainda, pela comparação dos valores do IR entre as diversas artérias avaliadas nos diferentes estágios do estudo, concluir serem as artérias cerebrais anteriores representativas das alterações máximas que ocorrem na resistência vascular cerebral em pacientes pediátricos portadores de hidrocefalia.
\end{abstract}

PALAVRAS-CHAVE: hidrocefalia, neurosonografia, Doppler craniano, índice de resistência, artérias intracranianas.

\section{Transfontanellar Doppler ultrasound measurement of cerebral blood velocity before and after surgical treatment of hydrocephalus}

ABSTRACT - Twenty-seven children with hydrocephalus of different etiologies diagnosed by clinical examination, neurosonography and computerized brain tomography were submitted to transfontanellar US-Doppler evaluation for measurement of blood flow velocity and for the calculation of resistance index (RI) in the anterior and middle cerebral arteries and internal carotids. All children were submitted to evaluation before surgery and on the 1st, 30th and 60th postoperative days. We conclude that neurosonography and US-Doppler technique is useful for determination of hydrocephalus, indication and control of cerebrospinal fluid shunts and monitoring of changes in RI, comparing data obtained immediately before and after surgery and during the late postoperative period. The results obtained when comparing the RI values for the various arteries during the different stages of the study also permitted us to conclude that the anterior cerebral arteries are representative of the maximal alterations that occur in cerebral vascular resistance in pediatric patients with hydrocephalus.

KEY WORDS: hydrocephalus, neurosonography, cranial Doppler, resistance index, intracranial arteries.

A hidrocefalia é patologia caracterizada pelo aumento do volume de líquor intraventricular e/ ou no espaço subaracnóideo. A incidência de hidrocefalia congênita sem espinha bífida tem sido

*Professor Assistente do Departamento de Cirurgia da Faculdade de Medicina da Universidade Federal de Uberlândia; **Professor Associado do Departamento de Cirurgia, Ortopedia e Traumatologia (Disciplina de Neurocirurgia) da Faculdade de Medicina de Ribeirão Preto da Universidade de São Paulo. Aceite: 28-junho-1999.

Dr. Marcelo Cardoso de Assis - Departamento de Cirurgia, Campus Umuarama, Universidade Federal de Uberlandia - 38701-136 Uberlandia MG - Brasil. 
estimada entre 1,2 a 15 casos por 10000 nascimentos ${ }^{1}$. O diagnóstico da hidrocefalia, amparado nas evidências clínicas, compreende hoje a utilização da ultra-sonografia (neurosonografia, NSG), tomografia computadorizada (TC) e ressonância magnética nuclear. A utilização da NSG em crianças com fontanela aberta que será utilizada como janela acústica tem sido amplamente descrita e difundida ${ }^{2-6}$ mostrando ser técnica eficaz e segura no diagnóstico e acompanhamento das hidrocefalias, revestindo-se ainda de caráter de extrema benignidade ao evitar expor o cérebro imaturo dos recémnascidos e lactentes à radiacão, podendo ser repetida sucessivas vezes e dispensando o uso de sedação para realização do exame.

Diversos estudos têm demonstrado a ocorrência de relação direta entre os vasos intracranianos e hidrocefalia. Hochwald e col. ${ }^{7}$ demonstraram que a hidrocefalia aguda produzida em gatos promove redução de $22 \%$ no fluxo sanguíneo do cérebro, cerebelo e tronco encefálico. Milhorat ${ }^{8}$ cita as várias alterações que ocorrem na circulação cerebral em decorrência de hidrocefalia enfatizando a redução do fluxo sanguíneo cerebral pelo aumento da pressão intracraniana bem como pelo estiramento e estreitamento das artérias cerebrais.

Até um passado recente o ultra-som Dopller (US-Doppler) não era utilizado para exame dos vasos intracranianos, pois acreditava-se que as ondas do ultra-som seriam atenuadas pelo crânio. Bada $^{9}$ foi quem primeiro avaliou as artérias intracranianas em neonatos utilizando o US-Doppler ${ }^{9}$. Aaslid ${ }^{10}$ determinou a velocidade de fluxo nos vasos do polígono de Willis utilizando US-Doppler transcraniano e quantificando valores para as artérias cerebral anterior, media e posterior, separadamente ${ }^{10}$. Pourcelot foi quem primeiro descreveu um índice de resistência vascular cerebral que correlacionava a amplitude sistólica e a amplitude diastólica final, minimizando assim os efeitos da variação do angulo do feixe ultra-sônico em relação à artéria estudada ${ }^{11,13}$. A partir de 1982, com a publicação dos estudos de Hill e Volpe ${ }^{14}$, surgiram vários trabalhos procurando determinar as alterações do fluxo pulsátil nas artérias cerebrais utilizando a técnica de US-Doppler em crianças portadoras de hidrocefalia. As pesquisas instituídas objetivaram a determinação do "timing" para cirurgia $^{13}$, observar o comportamento do índice de resistência (IR) em relação ao aumento da dilatação ventricular ${ }^{15}$, determinar a necessidade de shunt ventrículo-peritoneal através da evolução sequencial do $\mathrm{IR}^{16}$ e diagnosticar o funcionamento inadequado dos sistemas de derivação liquórica ${ }^{17}$.

Nossos objetivos ao realizar este estudo foram:

1) Determinar o IR nas artérias cerebrais anteriores direita (ACAD) e esquerda (ACAE), cerebrais médias direita (ACMD) e esquerda (ACME) e carótidas internas direita (ACID) e esquerda (ACIE) no pré e pós-operatório do tratamento cirúrgico da hidrocefalia em crianças, analisando as modificações na velocidade de fluxo sanguíneo cerebral.

2) Comparar entre si os valores do IR nas ACAD, ACAE, ACMD, ACME, ACID e ACIE no pré e pós-operatório do tratamento cirúrgico da hidrocefalia em crianças, observando seu comportamento.

3) Acompanhar a evolução da velocidade de fluxo sanguíneo e consequente IR utilizando US-Doppler no pós-operatório imediato e tardio da cirurgia para tratamento da hidrocefalia em crianças.

\section{MÉTODO}

No período compreendido entre maio de 1991 e junho de 1993, 27 crianças portadoras de hidrocefalia de diversas etiologias foram examinadas e acompanhadas conforme protocolo estabelecido. Quatorze crianças eram do sexo masculino $(51,9 \%)$ e 13 do sexo feminino $(48,1 \%)$. A faixa etária dos pacientes variou de 7 dias a 8 meses e 13 dias.

Os pacientes incluídos neste estudo submeteram-se ao seguinte protocolo:

1. Avaliação pediátrica e neurológica em que eram enfatizadas as medidas do perímetro craniano, inspecção e palpação da fontanela anterior (bregmática). Foram excluídas do estudo as crianças portadoras de patologias que sabidamente interferem na média da velocidade do fluxo sanguíneo ao Doppler tais como a persistência do 
ducto arterioso, pneumotórax, policitemia com hiperviscosidade ${ }^{18}$.

2. NSG: para diagnóstico e acompanhamento da dilatação ventricular através da determinação do índice ventricular, da espessura do córtex cerebral e do diâmetro látero-lateral do III ventrículo.

3. TC: todas as crianças nas quais a avaliação pela NSG não permitia o diagnóstico etiológico seguro da hidrocefalia ou ainda naqueles casos em que a NSG demonstrava hidrocefalia com outras malformações associadas.

4. Líquor ventricular e lombar: naquelas crianças cuja hidrocefalia era secundária a infecção do sistema neural já consideradas cliníca e laboratorialmente curadas realizávamos, 24 horas antes da cirugia de derivação liquórica, novo estudo do líquor ventricular e lombar. Naquelas crianças cuja hidrocefalia era secundária a disrafismo crânio-espinal realizávamos estudo do líquor ventricular 24 horas antes da cirurgia de derivação liquórica. Estando normal, indicava-se o tratamento cirúrgico.

5. US-Doppler transfontanela: utilizamos aparelhagem de ultra-som bidimensional com Doppler pulsátil (US-Doppler), cursor de 3,5; 5,0 ou 7,5 MHz, modelo Siemens SL-2 ( Siemens Aktienfessllschaft Erland, RDA).

Técnica.

O paciente (criança) é colocado sobre a mesa de exames em decúbito dorsal contida manualmente pela mãe ou acompanhante, sem utilização de qualquer sedação. Utilizamos a fontanela anterior como janela acústica e realizamos inicialmente o estudo de NSG das estruturas intracanianas. Identificamos o sistema ventricular, determinamos a espessura do córtex cerebral, o índice ventricular, o diâmetro látero-lateral do III ventrículo e nos certificamos da existência ou não de lesões extra-axiais ou intraparenquimatosas encefálicas. Passamos então a localizar os vasos intracranianos: ACID e ACIE, ACMD e ACME, ACAD e ACAE. Todos estes vasos são identificados mediante estudo em cortes coronais ao ultra-som sendo que as artérias cerebrais anteriores localizam-se sobre o corpo caloso, as artérias cerebrais médias a nível da fissura de Sylvius e as artérias carótidas internas lateralmente à sela túrcica.

Realizada a identificação dos vasos intracranianos estes foram estudados separadamente com registro de velocidade de fluxo pelo Doppler sendo consideradas as velocidades de fluxo sistólico e de fluxo diastólico final. Estas velocidades foram obtidas pela sua determinação por 5 a 20 vezes consecutivas computando-se uma média

O cálculo do IR foi realizado utilizando-se a expressão matemática:

$\mathrm{IR}=(\mathrm{S}-\mathrm{D}) / \mathrm{S}$

onde: $\mathrm{S}=$ velocidade de pico sistólico

$\mathrm{D}=$ velocidade diastólica final

dos valores. Posteriormente estes dados foram utilizados para o cálculo do índice de resistência.

\section{Determinação do índice de resistência (IR)}

Os dados da velocidade de pico sistólico e velocidade diastólica final foram obtidos através de medidas nas curvas de fluxo fornecidas pelo Doppler, cuja unidade é $\mathrm{cm} / \mathrm{s}$, sendo que o aparelho permite obter estes valores diretamente destas curvas.

Consideramos como valores normais do IR aqueles determinados por Hill e Volpe ${ }^{14}$, ou seja, variam de 0 a 1,0 com média de $0,66 \pm 0,06$.

\section{Tratamento Cirúrgico}

Todas as crianças foram submetidas a tratamento cirúrgico da hidrocefalia mediante a implantação de sistema de derivação ventrículo peritoneal (DVP).

\section{RESULTADOS}

Os valores dos índices de resistência obtidos nas artérias estudadas, nas diversas fases do estudo, encontram-se nas Figuras 1,2,3,4,5,6.

Em nosso estudo apesar de nos propormos a obter o IR nas seis artérias intracranianas isto nem sempre foi possível por motivos de ordem técnica, a saber: um paciente apresentava holoprosencefalia do tipo alobar sendo possível avaliar o fluxo ao Doppler somente nas artérias cerebrais médias e carótidas internas, pois as artérias cerebrais anteriores não foram identificadas; outro paciente apresentava hidrocefalia secundária a cisto de Dandy-Walker associado a cavidade 
Fig 1. Valores do IR na artéria cerebral anterior direita nos diferentes estágios de estudo.

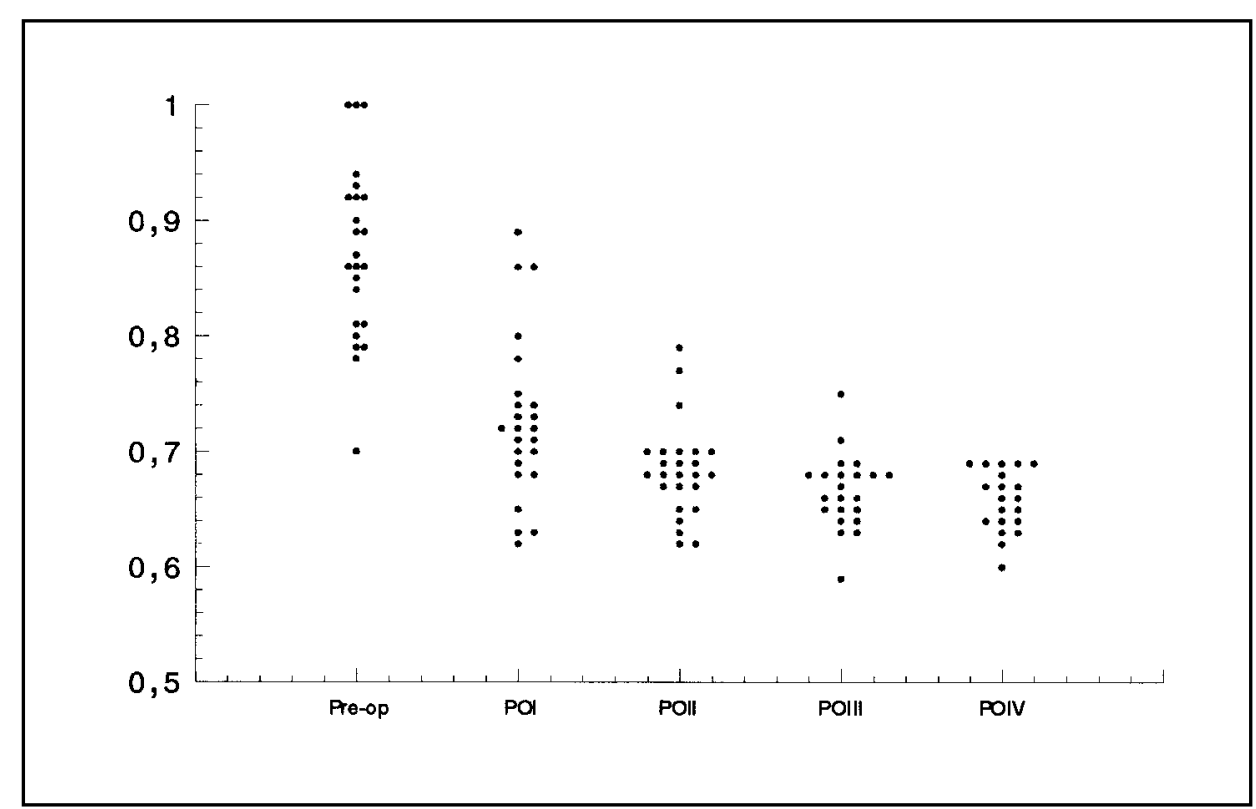

Pré-op., avaliação realizada no dia anterior à cirurgia; POI, avaliação realizada no $1^{o}$ dia pós-operatório; POII, avaliação realizada no $7^{\circ}$ dia pós-operatório; POIII, avaliação realizada no $30^{\circ}$ dia pós-operatório; POIV, avaliação realizada no $60^{\circ}$ dia pós-operatório

Fig 2. Valores do IR na artéria cerebral anterior esquerda nos diferentes estágios de estudo.

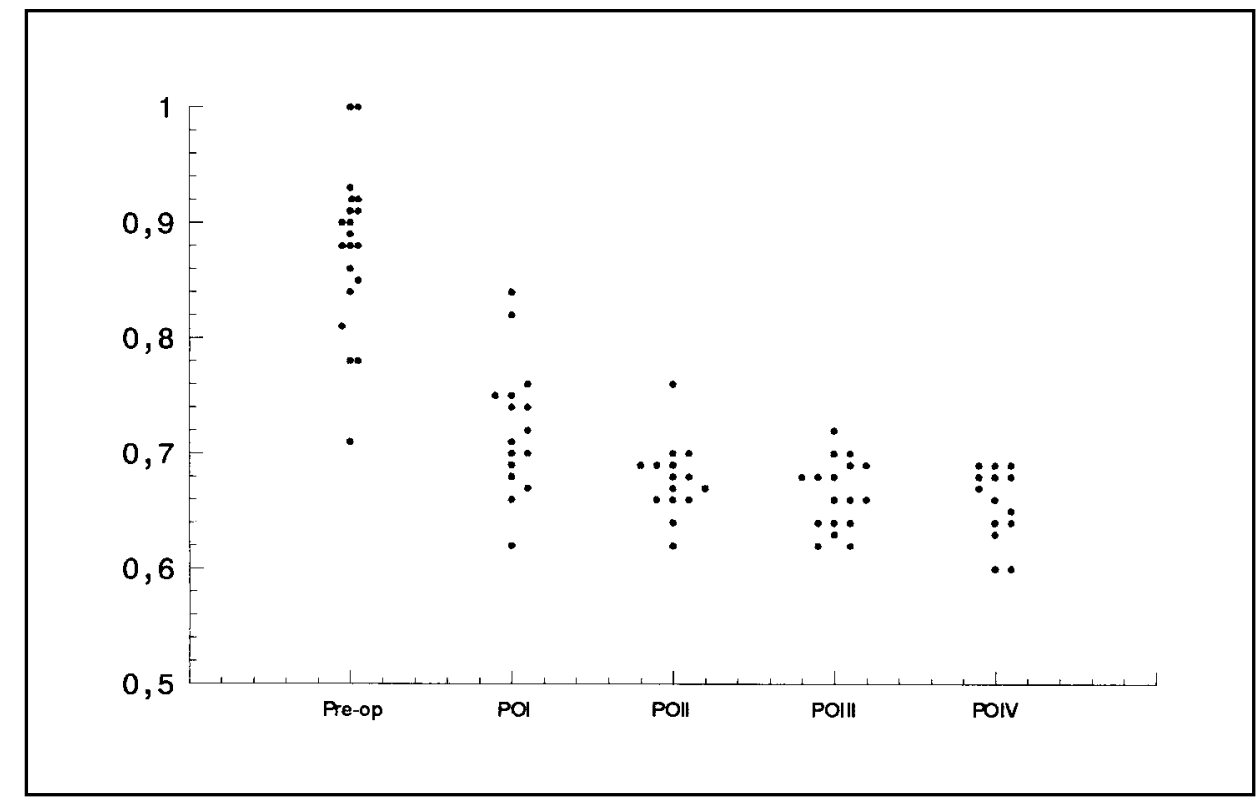

Legenda: Ver Fig 1. 
Fig 3. Valores do IR na artéria cerebral média direita nos diferentes estágios de estudo.

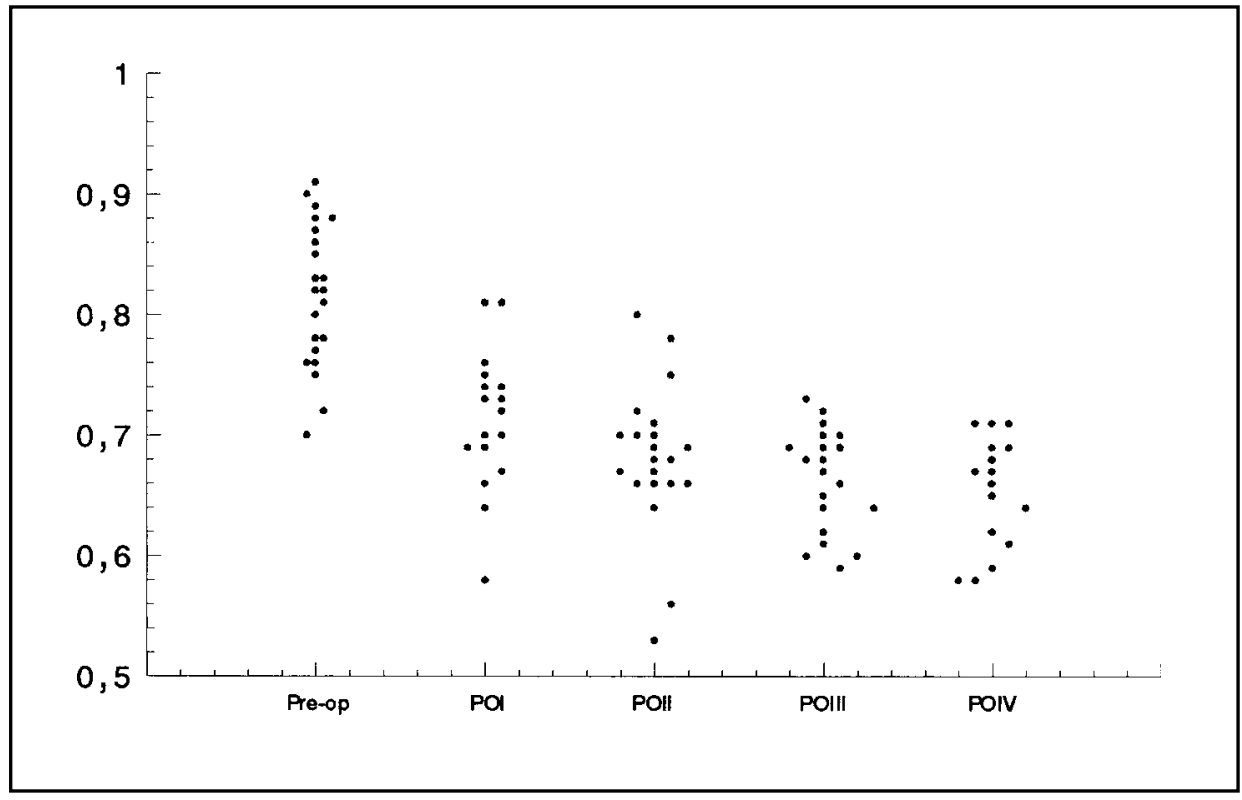

Legenda: Ver Fig 1.

Fig 4. Valores do IR na artéria cerebral média esquerda nos diferentes estágios de estudo.

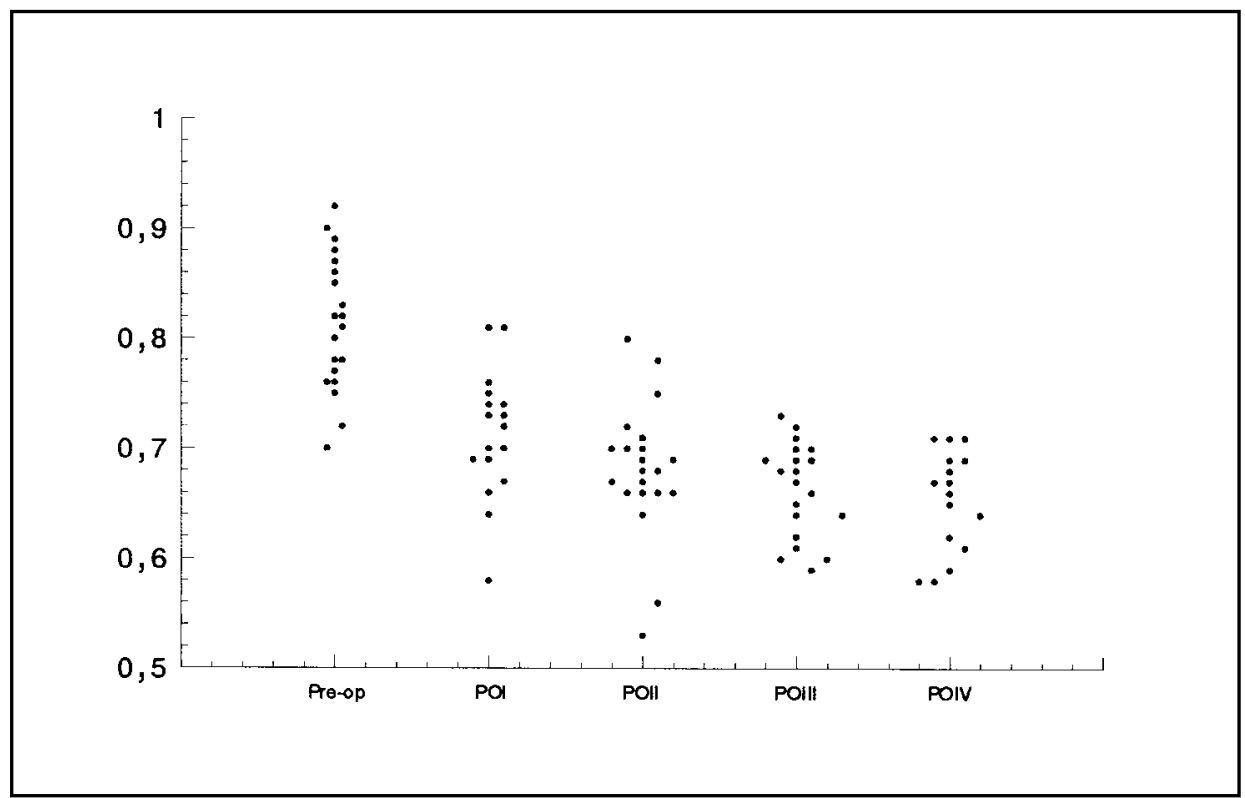

Legenda: Ver Fig 1. 
Fig 5. Valores do IR na artéria carótida interna direita nos diferentes estágios de estudo.

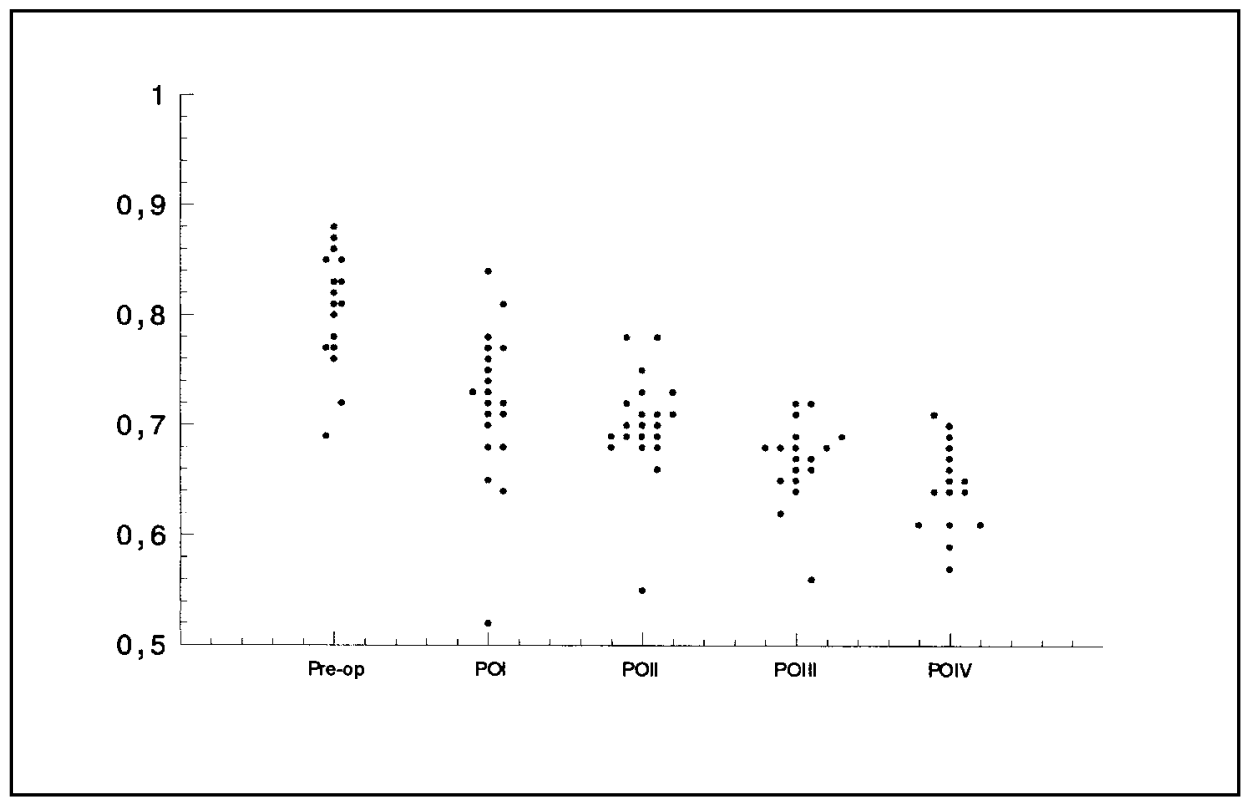

Legenda: Ver Fig 1.

Fig 6. Valores do IR na artéria carótida interna esquerda nos diferentes estágios de estudo.

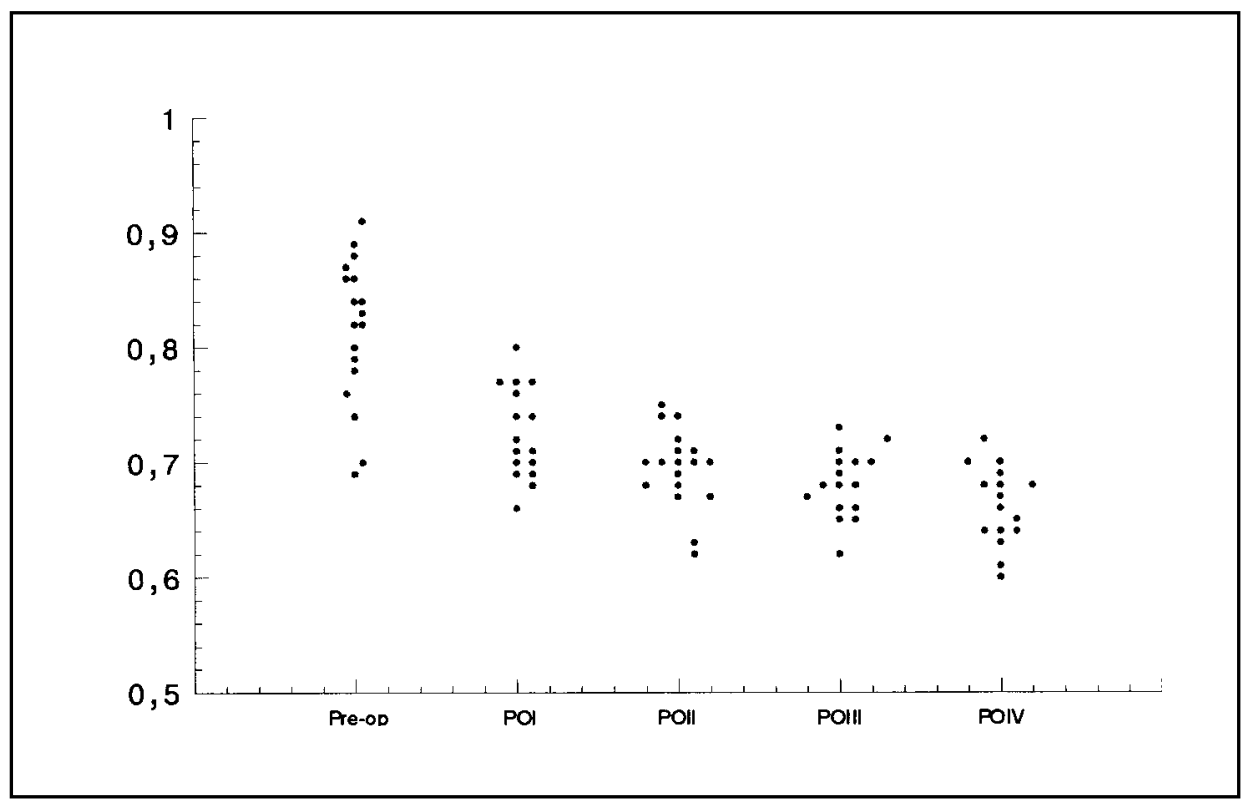

Legenda: Ver Fig 1. 
ventricular única supratentorial não sendo possível determinar a velocidade de fluxo nas artérias cerebrais anteriores, pois elas não foram identificadas à NSG.

Análise Estatística - Na análise estatística foi utilizado o teste t pareado com intervalo de confiança. Este teste comparou cada artéria avaliada em cada fase do estudo.

A comparação dos IR na ACAD no pré e pós-operatório I mostrou queda do IR estatisticamente significante $(\mathrm{p}<0,05)$.

A comparação dos IR no pré e pós-operatório II mostrou queda do IR mais acentuada que aquela observada entre o pré e o pós-operatório I ( $\mathrm{p}<0,05$ ). A comparação dos IR no pré e pósoperatório III e IV mostrou também queda significativa do IR.

A análise estatística demonstrou que as avaliações dos IR nas artérias ACMD, ACME, ACID e ACIE apresentaram o mesmo comportamento que aquele observado nas artérias cerebrais anteriores, nas diversas fases do estudo.

Entretanto a comparação das artérias entre si demonstrou que as variações do IR foram mais significantes na artéria cerebral anterior indicando-nos ser esta artéria mais sensível às modificações da velocidade de fluxo sanguíneo nas diversas fases do estudo.

\section{DISCUSSÃO}

A utilização da NSG para diagnóstico e acompanhamento do tratamento cirúrgico da hidrocefalia cada vez mais tem sido incorporado à prática neurocirúrgica, sobretudo da neurocirurgia pediátrica bem como da neuropediatria, da neonatologia e da pediatria. A NSG, além de consideradas todas as suas características de benignidade como recurso propedêutico, permite determinar a etiologia da hidrocefalia, classificar o seu grau fornecendo avaliações sobre a espessura do córtex cerebral, diâmetro das cavidades ventriculares e o índice ventricular. Nos últimos anos alguns estudos foram publicados abordando a técnica de US-Doppler em hidrocefalia. Hill e Volpe ${ }^{14}$ estudaram crianças portadoras de hidrocefalia e realizaram avaliação por US-Doppler transfontanela com medida do IR na artéria cerebral anterior antes e após o tratamento cirúrgico da ventriculomegalia. Observaram decréscimo uniforme do IR nos pacientes submetidos a cirurgia. Em quatro pacientes, de onze avaliados, esses autores observaram IR máximo, ou seja, igual a 1,0.

Em nosso estudo, dos 27 pacientes avaliados, em três observamos IR igual a 1,0 e somente na avaliação obtida na artéria cerebral anterior, não tendo sido este valor máximo detectado em nenhuma outra artéria daquelas avaliadas, naquele momento. Acreditamos que a artéria cerebral anterior pela sua peculiaridade anatômica em relação ao sistema ventricular sofra mais intensamente as consequências da ventriculomegalia em que o estiramento e estreitamento vascular acarretariam desaparecimento do fluxo sanguíneo durante a fase de diástole levando, por conseguinte, a IR máximo.

Quando comparamos os valores do IR no pré-operatório e primeiro dia pós-operatório, em todas as artérias avaliadas, observamos queda significativa dele motivada pela descompressão ventricular. Esse dado permite ao neurocirurgião certificar-se da patência do sistema de derivação liquórica no pós-operatório imediato e realizar o acompanhamento subsequente possibilitando a detecção de disfunções deste sistema.

A comparação do IR no pré-operatório com o $7^{\circ}$ dia pós-operatório mostrou queda significativa dele mais acentuada que aquela observada entre o pré-operatório e o $1^{\circ}$ dia pós-operatório. A partir do $30^{\circ}$ dia pós-operatório observamos estabilização do IR não havendo queda dele quando comparados o $30^{\circ}$ com o $60^{\circ}$ dias do pós-operatório.

Nosso estudo demonstrou que a queda do IR ocorre em função de um acréscimo da velocidade diastólica final (VDF) e não às custas de decréscimo da velocidade de pico sistólico (VS). A este respeito existem dados conflitantes na literatura pertinente. Alvisi e col. ${ }^{19} \mathrm{e}$ Van Bell e col. ${ }^{20}$ relatam que a modificação do IR ocorre devido a queda da VS não ocorrendo modificações significativas 
dos valores da VDF. Outros estudos entretanto demonstram exatamente o contrário como aqueles de Hill e Volpe ${ }^{14}$, Goh e col. ${ }^{21}$ e Huang e Chio ${ }^{22}$ que constataram quedas do IR por acréscimo da VDF o que significaria uma diminuição da resistência vascular cerebral.

Quando comparamos o IR e suas variações, nas diversas fases do estudo, entre as artérias avaliadas - ou seja, IR na ACAD e ACAE comparados ao IR na ACMD e ACME; IR nas ACAD e ACAE comparados ao IR na ACID e ACIE; IR na ACMD e ACME comparados ao IR na ACID e ACIE - observamos que as variações dos valores do IR foram mais acentuados na artéria cerebral anterior demonstrando-nos ser esta artéria mais sensível às modificações da velocidade de fluxo sanguíneo nas diversas etapas do estudo. Acreditamos que quando da realização de US-Doppler, para medida da velocidade de fluxo em hidrocefalia e determinação do IR, a avaliação realizada somente na artéria cerebral anterior será fidedigna quanto às alterações máximas que estejam ocorrendo na resistência vascular cerebral daquele paciente hidrocéfalo.

Ainda segundo os trabalhos de Hill e Volpe ${ }^{14}$, Alvisi e col. ${ }^{19}$ Chadduck e col. ${ }^{16}$, Goh e col. ${ }^{21} \mathrm{e}$ Huang e $\mathrm{Chio}^{22}$, a utilização da técnica de US-Doppler com evolução sequencial do IR, usada em conjunto com as observações de caráter clínico é extremamente útil e pode predizer a necessidade de indicação de shunt de derivação liquórica.

Estes dados da literatura puderam ser plenamente observados em vários de nossos pacientes nos quais notamos, por avaliações sucessivas do IR no pré-operatório, elevação gradativa dele chegando em alguns pacientes ao valor máximo de 1,0. A indicação de cirurgia para estes pacientes, observados os critérios clínico e da NSG, levou-nos a observar queda significativa do IR no pósoperatório imediato demonstrando o que foi observado pelos autores citados anteriormente.

Chadduck e col. ${ }^{17}$ estudaram 41 pacientes com funcionamento inadequado do sistema de derivação liquórica usando a técnica de Doppler transcraniano. Este funcionamento inadequado era caracterizado por evidência clínica e radiográfica do aumento da pressão intracraniana. Nestes 41 pacientes foi realizado determinação do IR antes e após revisão do sistema de derivação liquórica. Os autores observaram IR de 0,71 $\pm 0,10$ antes da revisão do sistema que caíram para 0,53 $\pm 0,10$ após a revisão dos mesmos. Estes dados, estatisticamente significantes, demonstraram para os autores uma correlação entre elevação do IR e funcionamento inadequado do shunt.

Durante nosso estudo dois pacientes apresentaram obstrução do sistema de derivação liquórica. A primeira criança apresentou, 48 dias após a realização da cirúrgia inicial de derivação liquórica, sinais de irritabilidade, fontanela anterior abaulada e o estudo com US-Doppler mostrava elevação do IR. Foi então realizada revisão cirúrgica do sistema de DVP encontrando-se obstrução do catéter distal por aderência peritoneal e no pós-operatório imediato houve queda acentuada do IR bem como melhora clínica da criança.

Uma segunda criança apresentou, 54 dias após a realização da cirurgia inicial de DVP manifestações clínicas de torpor, fontanela anterior abaulada e crises convulsivas. Estudo NSG com Doppler mostrou IR elevado. Foi realizada revisão do sistema de DVP, encontrando-se obstrução do catéter ventricular. No primeiro dia pós-operatório a criança encontrava-se alerta, fontanela anterior normotensa e o IR que de 0,92 antes da revisão da DVP caiu para 0,52 após a troca do catéter ventricular, valores estes medidos na artéria cerebral anterior direita e esquerda.

Estes dois casos ilustram, em nosso estudo, a sensibilidade do US-Doppler em detectar através dos valores do IR o funcionamento inadequado do shunt de derivação liquórica e sua ulterior normalização.

\section{CONCLUSÕES}

Os resultados obtidos do estudo de 27 pacientes portadores de hidrocefalia utilizando NSG e US-Doppler no pré e pós-operatório do tratamento cirúrgico desta patologia mostram a validade desta técnica não invasiva na indicação e controle das derivações liquóricas. 
Nas avaliações pós-operatórias observamos que a medida da velocidade de fluxo com consequente determinação do IR permite ao neurocirurgião certificar-se, já no primeiro dia póscirurgia, da patência e do funcionamento adequado do sistema de derivação liquórica. Permite ainda, pelas avaliações subsequentes, diagnosticar o funcionamento inadequado do sistema de derivação liquórica pela comparação do IR no pré e pós-operatório imediato e tardio com os valores obtidos quando da suspeita clínica de disfunções do sistema de derivação liquórica.

A comparação dos valores do IR entre as diversas artérias, nas diversas fases do estudo, permitiu-nos concluir ser a artéria cerebral anterior um espelho fiel das alterações que ocorrem na resistência vascular cerebral podendo-se mesmo dispensar a avaliação sobre as demais artérias.

\section{REFERÊNCIAS}

1. McCullough JC. Hydrocephalus: etiology, pathologic effects, diagnosis and natural history In Epstein F. Pediatric neurosurgery, Philadelphia: W.B. Saunders, 1989:180-199.

2. Babcock DS, Han BK, Le Quesve GW. B-mode gray scale ultrasound of the head in newborn and young infant. AJR 1980;134:457-468.

3. Ben Ora A, Eddy L, Hatch G, Solida P. The anterior fontanelle as an acoustic window to the neonatal ventricular system. J Clin Ultras 1980;8:65-67.

4. Machado HR, Machado JC, Contrera J D, Assirati JA Jr, Martelli, N. Ultrasonografia cerebral em crianças no primeiro ano de vida: um método não invasivo para o diagnóstico e acompanhamento das dilatações ventriculares. Arq Neuropsiquiatr1982;40:385-389.

5. Machado HR, Machado JC, Contrera, JD Ultrasonographic evaluation of infantile hydrocephalus before and after shunting. Child's Nerv Syst 1985;1:341-344.

6. Schackelford GD Neurosonography of hydrocephalus in infants. Neuroradiology 1985;28:452-462.

7. Hochwald GM, Bolad RD, Marlin A, Kumar AJ Changes in regional blood flow and water content of brain and spinal cord in acute and chronic experimental hydrocephalus. Dev Med Child Neurol 1975;35(Suppl):42-50.

8. Milhorat TG Hydrocephalus and the cerebrospinal fluid. Baltimore: William and Wilkins, 1972.

9. Bada HS, Hassar W, Chua, C, Sumner DS. Noinvasive diagnosis of neonatal asphyxia intraventricular hemorrhage by Doppler ultrasound. J.Pediatr 1979;95:775-779.

10. Aaslid R, Markwalder TM, Normes H. Noninvasive transcranial Doppler ultrasound recordings of flow velocity in basal cerebral arteries. J Neurosurg. 1982;57:769-774.

11. Pourcelot L. Diagnostic ultrasound for cerebral vascular diseases. In Dover D. Present and future of diagnostic ultrasound. Rotterdam: Kooyer, 1976:141-147.

12. Pourcelot L. Examen Doppler de la circulaction cerebrale. In Itti R, Pottier JM, Pourcelot L. Les explorations cardiovasculaires. Paris:INSERM, 1980;283-318.

13. Pourcelot L, Saliba E, Santini JJ Mesure non invasive du flux sanguin cerebral chez le nouveanune hydrocephale Neurochirurqie 1982;31:7-13.

14. Hill A. Volpe JJ Decrease in pulsatile flow in the anterior cerebral arteries in infantile hydrocephalus Pediatrics 1982;69:4-7.

15. Lui K, Hellmann J, Sprigg A, Daneman, A. Cerebral blood flow velocity patterns in post-hemorrhagic ventricular dilation. Child's Nerv Syst 1990;6:250-253.

16. Chadduck WM, Crabtree HM, Blanskenship JB. Transcranial Doppler ultrasonography for the evaluation of shunt malfunction in pediatric patients. Child's Nerv Syst 1991;7:27-30.

17. Chadduck WM, Seiberg JJ, Adametz, J. Cranial Doppler ultrasonography correlates with criteria for ventriculoperitoneal shunting. Surg Neurol 1989;31:122-128.

18. Seibert JJ, McCowan TC, Chadduck WM Duplex pulsed Doppler US versus intracranial pressure in the neonate: clinical and experimental studies Radiology 1989;171:155-159.

19. Alvisi C, Cerisoli M, Giulioni M. Evaluation of cerebral blood flow changes by transfontanelle Doppler Ultrasound in infantile hydrocephalus. Child's Nerv Syst 1985;1:244-247.

20. Van Bell F, Van de Bor M, Baan J. Blood flow velocity pattern of the anterior cerebral arteries before and after drainage of posthemorrhagic hydrocephalus in the newborn. J Ultrasound Med 1988;7:553-559.

21. Goh D, Minns RA, Dye SD, Steers AJN. Cerebral blood flow velocity changes after ventricular taps and ventriculoperitoneal shunting. Child's Nerv Syst 1991;7:452-457.

22. Huang CC, Chio CC Duplex color ultrasound study of infantile progressive ventriculomegaly. Child Nerv's Syst 1991;7:251-256. 